\section{<P-2-18-3 $>$}

Docking protein Gab1 is essential for postnatal angiogenesis via hepatocyte growth factor (HGF)/c-Met signaling pathway

Wataru Shioyama ${ }^{1}$, Yoshikazu Nakaoka ${ }^{1}$, Kaori Higuchi ${ }^{1}$, Takashi Minami $^{2}$, Yoh Arita ${ }^{1}$, Hiroyasu Kidoya ${ }^{3}$, Hisamichi Naito ${ }^{3}$, Yoshiaki Taniyama ${ }^{4}$, Nobuyuki Takakura ${ }^{3}$, Ryuichi Morishita ${ }^{4}$, Yasushi Fujio ${ }^{1}$, Keiko Yamauchi-Takihara ${ }^{1}$, Toshio Hirano ${ }^{5}$, Tatsuhiko Kodama ${ }^{2}$, Naoki Mochizuki ${ }^{6}$, Issei Komuro

Department of Cardiovascular Medicine, Osaka University Graduate School of Medicine ${ }^{1}$; Research Center for Advanced Science and Technology, University of $\mathrm{Tokyo}^{2}$; Department of Signal Transduction, Research Institute for Microbial Diseases, Osaka University ${ }^{3}$; Department of Clinical Gene Therapy, Osaka University Graduate School of Medicine; ${ }^{4}$ Laboratory of Developmental Immunology, Osaka University Graduate School of Medicine; Department of Structural Analysis, National Cardiovascular Center Research Institute ${ }^{6}$, Japan

Docking protein Gab1 coordinates downstream signaling pathways of various receptor tyrosine kinases. Here, we examined the role of Gab1 in the postnatal angiogenesis through the creation of endothelium-specific Gab1 knockout mice (Gab1ECKO). Gab1 ECKO did not show any obvious developmental vascular defects. Intriguingly, Gab1ECKO displayed severe limb necrosis after hindlimb ischemia (HLI), while control mice did not show limb necrosis. Among various angiogenic factors such as VEGF, FGF2, and hepatocyte growth factor (HGF), HGF stimulation induced the strongest tyrosine-phosphorylation of Gab1 and complex formation of Gab1 with tyrosine phosphatase SHP2 and PI3-kinase subunit p85 in HUVECs. While Gab1-SHP2 complex was required for activation of both ERK1/2 and ERK5, Gab1-p85 complex was required for activation of AKT after stimulation with HGF. Using DNA microarray, we found that Gab1-SHP2 complex was required for the upregulation of Kruppel-like factor 2 (KLF2), a crucial transcriptional factor for endothelial maintenance in HUVECs. Furthermore, MEK5-ERK5 pathway was involved in HGF-induced upregulation of KLF2. In addition, the expression of KLF2 in the endothelium was significantly decreased in the Gab1ECKO, compared with control mice after HLI. These findings indicate that Gab1 is essential for HGF-dependent endothelial signaling and postnatal angiogenesis after ischemia, presumably via activation of ERK5-KLF2 pathway.

\section{$<$ P-2-18-5 $>$}

The role of the innate immune system in the development of a collateral circulation

Wolfgang Schaper

Max Planck Society, Germany

It is known that physical forces outside the physiological range, can activate the innate immune system (IIS). This became known when it was shown that LPS, the classical activator of the IIS (via TNF-alpha and iNOS), is a stimulant of the growth of collateral arteries following an arterial occlusion. The physical force initiating collateral growth is chronically increased fluid shear stress driven by the pressure gradient along the occlusion-spanning arterioles. We tested the hypothesis whether augmented and prolonged increase of fluid shear stress by creating a shunt between the distal stump of the occluded artery with the accompanying vein would lead to increased growth of collateral vessels. This was the case and it led to completely normalized and overshooting function. Grossly enlarged collateral vessels were harvested and their RNA subjected to genome wide screening by micro-array and 360 differentially regulated transcripts where found. These could be classified into shear stress-sensitive genes, like klf2, ion-channels like TRPV4, actin binding proteins like Abra, Thymosin beta 4, destrin, cofilin, smoothelin and SMmyosin heavy chain, the NO-generating enzymes eNOS and iNOS, and transcription factors like carp. The pattern of expression was dominated by the phenotypic change of smooth muscle as it undergoes transformation from the contractile to the proliferative and synthetic phenotype. From our present and past studies the following sequence of events can be deduced: chronically increased shear stress leads to early activation of klf2, TRPV4 and eNOS, which activates VEGF production via NO to start endothelial proliferation. A so far poorly defined endothelial signal reaches the smooth muscular media and stimulates SM proliferation after above described phenotype change. Abra plays a dominant role because it is a Rho-activating protein with mitogenic properties and its inhibition blocks arteriogenesis. Shear stressed endothelium but also the transforming muscular media excrete MCP-1 leading to attraction and adhesion of monocytes that initiate positive remodeling of the collateral structure via activation of matrix metallo-proteinase and elastase, produce TNF-alpha and iNOS and create a peri-vascular inflammation. Genetic or pharmacologic inhibition of the IIS, via TNF-alpha, iNOS, MCP-1, collateral artery growth could be completely abolished. We conclude that arteriogenesis is dependent on the innate immune system when it is activated by a physical stimulus, e.g., fluid shear stress.

\section{$<$ P-2-18-4 $>$}

Identification of potential candidates for novel signaling molecules which mediate vasospasm by focused proteomics Shoko Nakajima ${ }^{1}$, Hiroko Kishi ${ }^{2}$, Hozumi Kawamichi ${ }^{2}$, Katsuko Kajiya ${ }^{2}$, Yuichi Takada ${ }^{2}$, Daisuke Tokumori ${ }^{2}$, Sei Kobayashi ${ }^{2}$

Fourth grade student, Yamaguchi University School of Medicine ${ }^{1}$; Department of Molecular Physiology and Medical Bioregulation, Yamaguchi University Graduate School of Medicine $^{2}$, Ube, Japan

Abnormal vascular smooth muscle contraction (vasospasm) is one of the crucial phenomena which cause cardiac angina and brain infarction. We previously found that sphingosylphosphorylcholine (SPC) activates Rho-kinase (ROK) through the activation of Fyn, a member of Src family tyrosine kinase, inducing abnormal vascular smooth muscle contraction. However, the target(s) of Fyn, which lead to the activation of ROK, are unidentified. In the present study, we attempted to identify potential candidates for the target(s) of Fyn, by focused proteomics. Since Fyn is supposed to tyrosine-phosphorylate the target molecule(s), the tyrosine-phosphorylated proteins were enriched from the SPC-stimulated human coronary artery smooth muscle cell lysates, using an immunoprecipitation with anti-phosphotyrosine antibody. Those tyrosine-phosphorylated proteins were separated by SDS-PAGE, transferred to PVDF membrane, and digested with LysC. We excised 59 protein bands from the PVDF membrane, and identified 21 kinds of proteins out of 17 bands, using nanoLC-tandem mass spectrometry. Those identified proteins included six cytoskeleton-related proteins, five transport-related proteins, and three enzymes. Further studies are needed to determine which one(s) truly activate ROK to induce vasospasm. We found that focused proteomic approach is useful to reveal possible targets of Fyn, and their functional roles are under investigation.

\section{$<$ P-2-18-6 $>$}

Effects of abacavir on the vascular and platelet activities RWS Li ${ }^{1}$, YW Kwan ${ }^{2}$ and GPH Leung

Department of Pharmacology and Pharmacy, The University of Hong Kong ${ }^{1}$; School of Biomedical Science, The Chinese University of Hong Kong ${ }^{2}$, China

Abacavir is an antiviral nucleoside analogue effectively used to treat HIV infection. However, a study showed that the use of abacavir significantly increased the risks of stroke and myocardial infraction. We sought to investigate the effects of abacavir on vascular and platelet activities so as to understand the underlying mechanisms. Sprague-Dawley rats (330-350 g) were fed with abacavir $(16 \mathrm{mg} / \mathrm{kg} /$ day, equivalent to the clinical dosage on human) for 28 days by gastric gavage. Isometric tensions of basilar arteries were measured. Aorta mRNA and proteins expressions of various factors related to endothelial function and inflammation were measured by RT-PCR and Western blotting, respectively. To measure the effect of abacavir on platelet activation, the plasma levels of CD40L, a platelet-derived factor which is released upon platelet activation, were measured by ELISA kit. Our results showed that abacavir treatment did not affect the maximum relaxation response of basilar artery. Besides, there were no significant change in the mRNA and protein expression levels of eNOS, COX-1, COX-2 and ICAM-1 in aorta after abacavir treatment. However, a higher plasma level of CD40L was detected in the abacavir-treated group. It is suggested that abacavir does not affect vascular contractility nor induced endothelial inflammation. However, abacavir upregulates the platelet activity, which will theoretically increase the chance of developing thrombosis. This may be a possible explanation for the higher risk of cardiovascular events in patients receiving abacavir treatment. 